\title{
Preparation of new biocomposite designed for cartilage grafting with anti- biofilm activity
}

\author{
Sara Targonska ${ }^{1}$, Justyna Rewak-Sorczynska ${ }^{1}$, Agata Piecuch², Emil Paluch ${ }^{3}$, Damian \\ Szymanski ${ }^{1}$ and Rafal J. Wiglusz ${ }^{1, *}$ \\ ${ }^{1}$ Institute of Low Temperature and Structure Research, Polish Academy of Sciences, Okolna 2, 50-422 Wroclaw, \\ Poland \\ ${ }^{2}$ Institute of Genetics and Microbiology, University of Wroclaw, Przybyszewskiego 63/77, 51-148 Wroclaw, Poland \\ ${ }^{3}$ Department of Microbiology, Faculty of Medicine, Wroclaw Medical University \\ Tytusa Chalubinskiego 4, 50-376 Wroclaw, Poland
}

Keywords: Polymer-inorganic composites; PTFE polymer; Nanoapatites; $\mathrm{Ag}^{+}$ions; Cytotoxicity; Antibacterial properties

\footnotetext{
*Corresponding authors:

E-mail: r.wiglusz@intibs.pl

Phone: +48(071)3954159

Fax: +48(071)3441029
} 


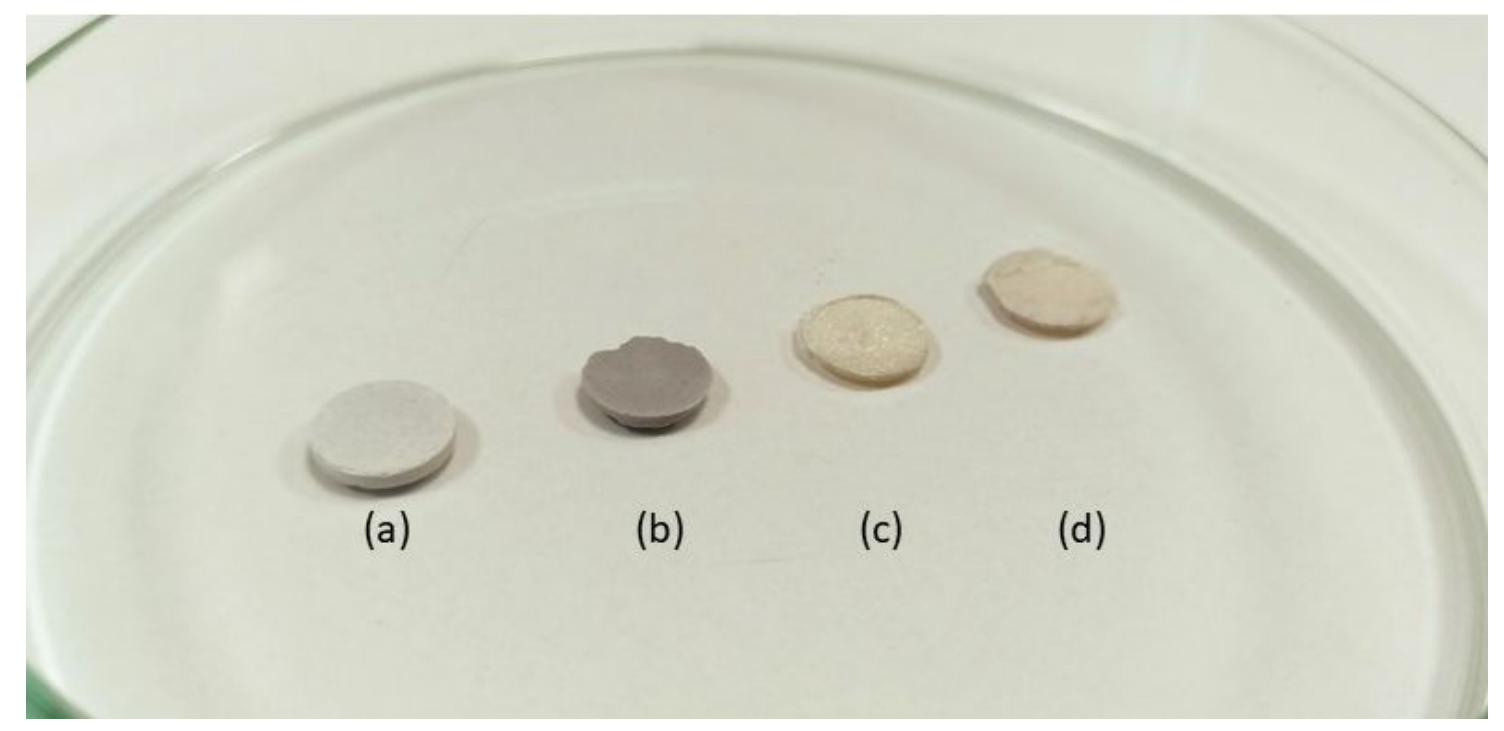

Figure S1. Representative pellets of (a) $5.0 \mathrm{~mol} \% \mathrm{Ag}^{+}: \mathrm{HAp}$; (b) $5.0 \mathrm{~mol} \% \mathrm{Ag}^{+}: \mathrm{FAp}$; and tetrafluoroethylene with (c) $10 \mathrm{wt} . \%$ of hydroxyapatite (PTFE@ $@ \mathrm{Ag}^{+}: 10 \mathrm{HAp}$ ) and (d) $10 \mathrm{wt} . \%$ of fluorapatite (PTFE@Ag+:10FAp) composites.
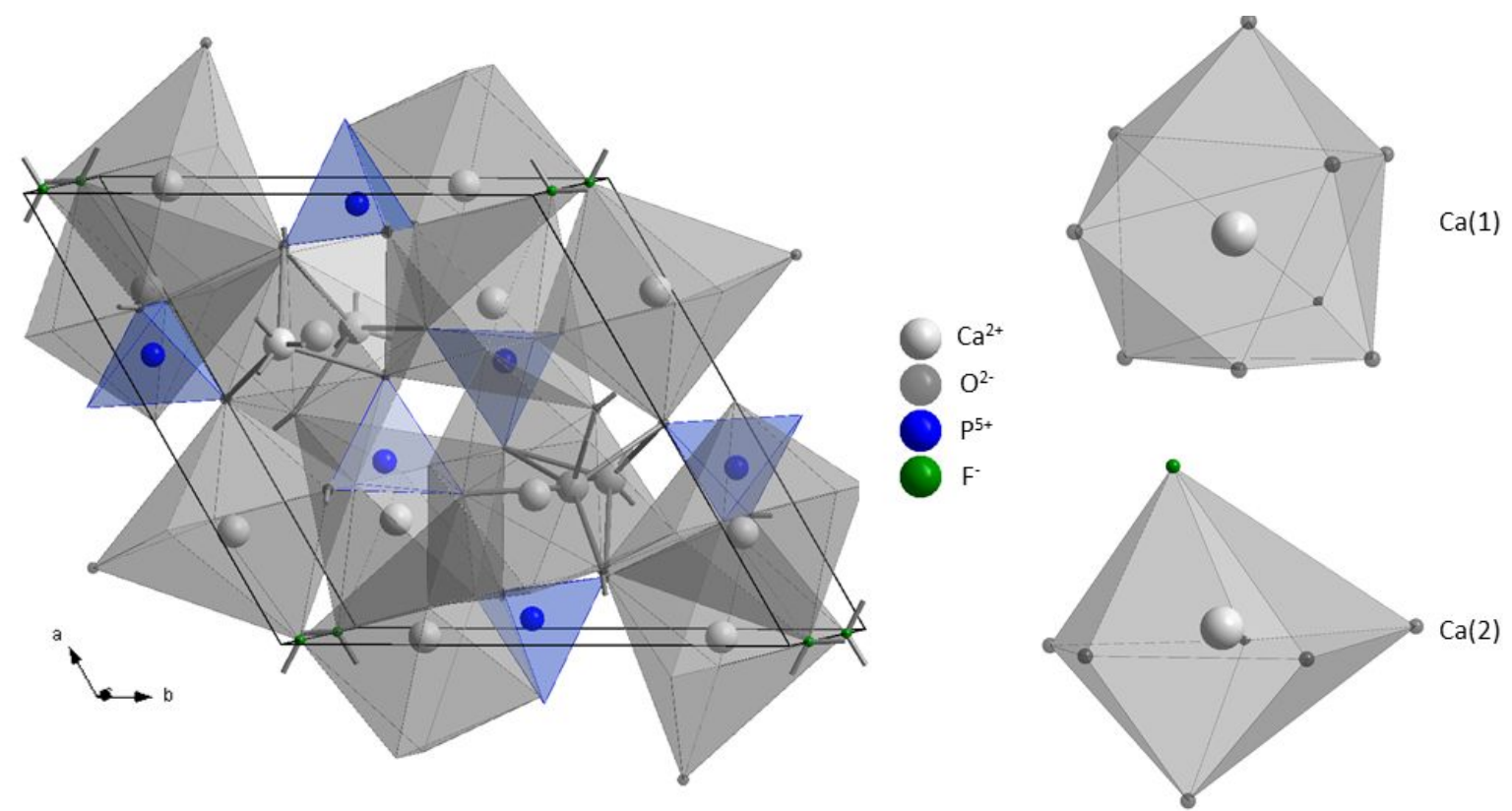

Figure S2. View of the unit cell of fluorapatite crystal along [001] and coordination polyhedron of $\mathrm{Ca}^{2+}$ cations in the crystal structure of the fluorapatite. 


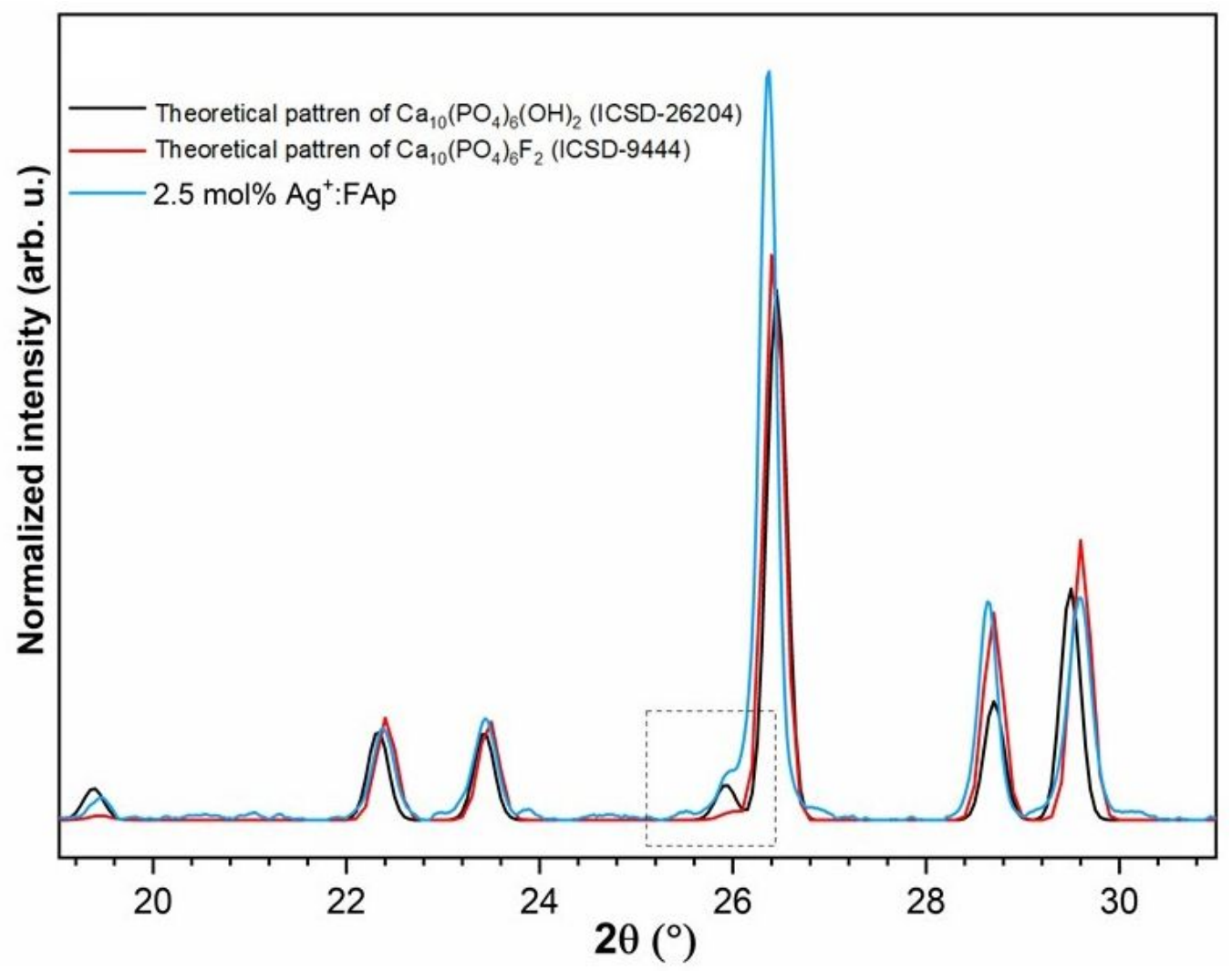

Figure S3. X-ray diffraction correlation of $2.5 \mathrm{~mol} \% \mathrm{Ag}^{+}: \mathrm{FAp}$ with hydroxyapatite and flourapatite.

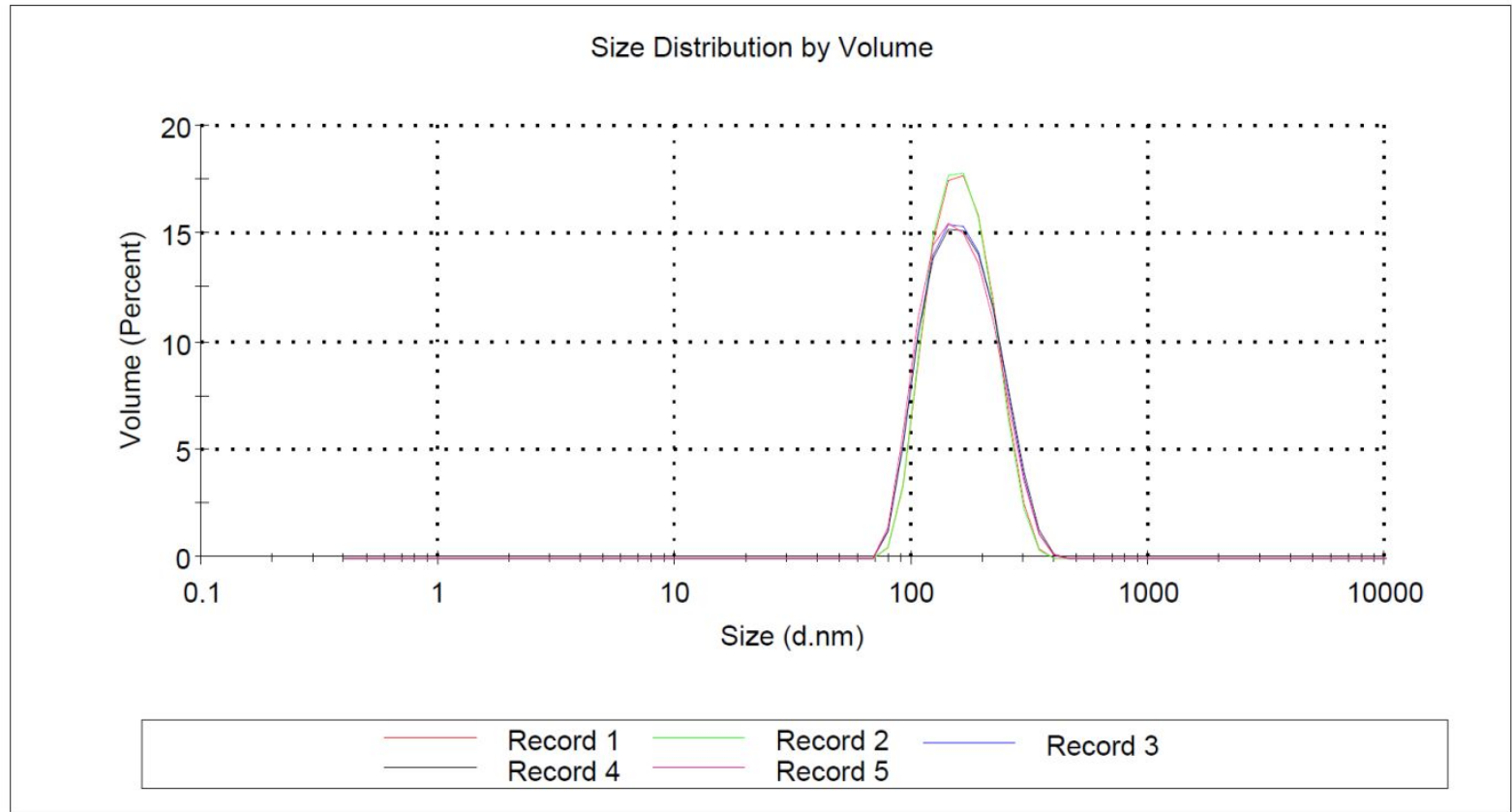

Figure S4. The particle size distribution of dispersed poly(tetrafluoroethylene) PTFE in deionized water. 


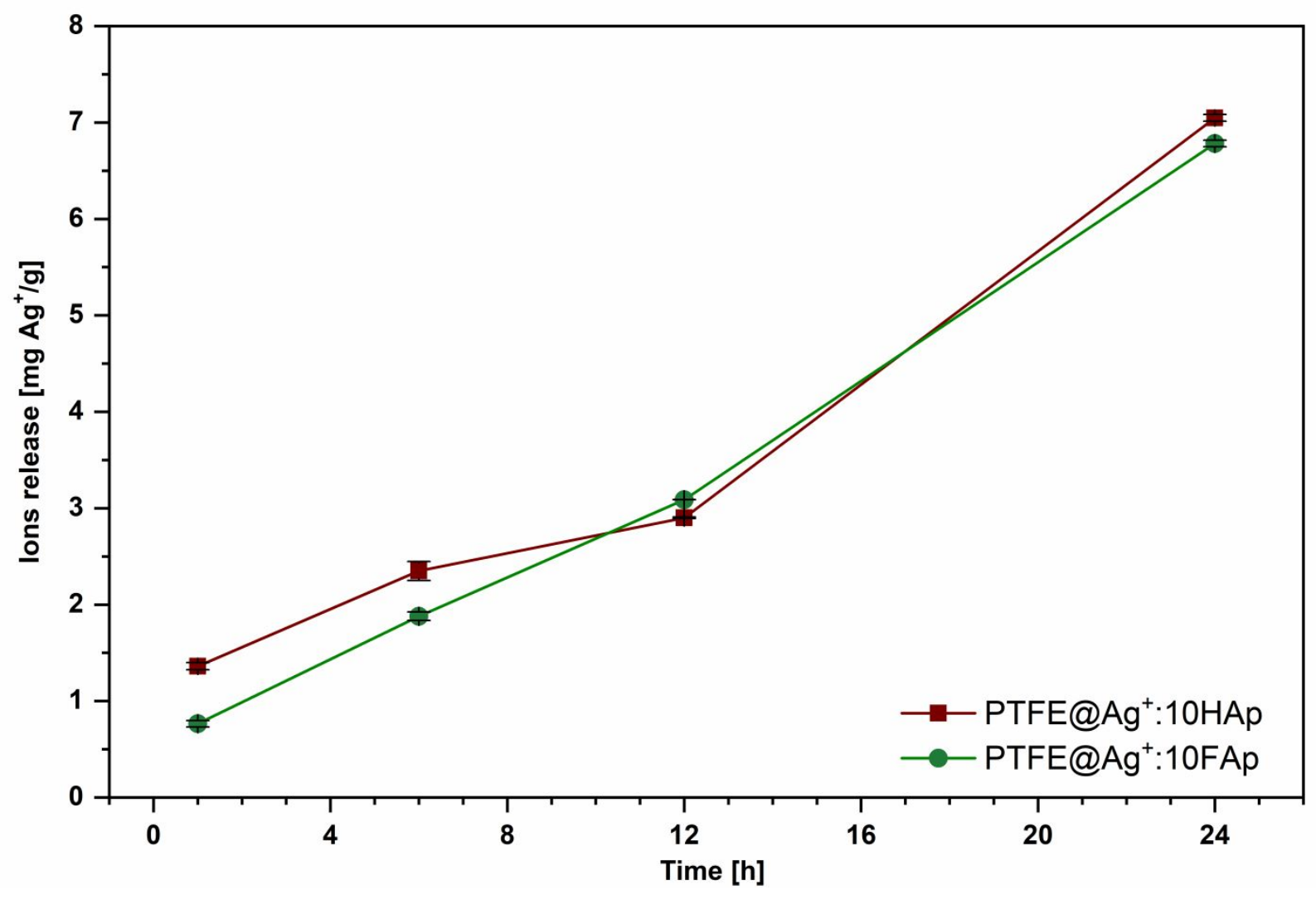

Figure S5. The $\mathrm{Ag}^{+}$release from tetrafluoroethylene composites with $10 \mathrm{wt} . \%$ of hydroxyapatite and 10 wt.\% of fluorapatite. 


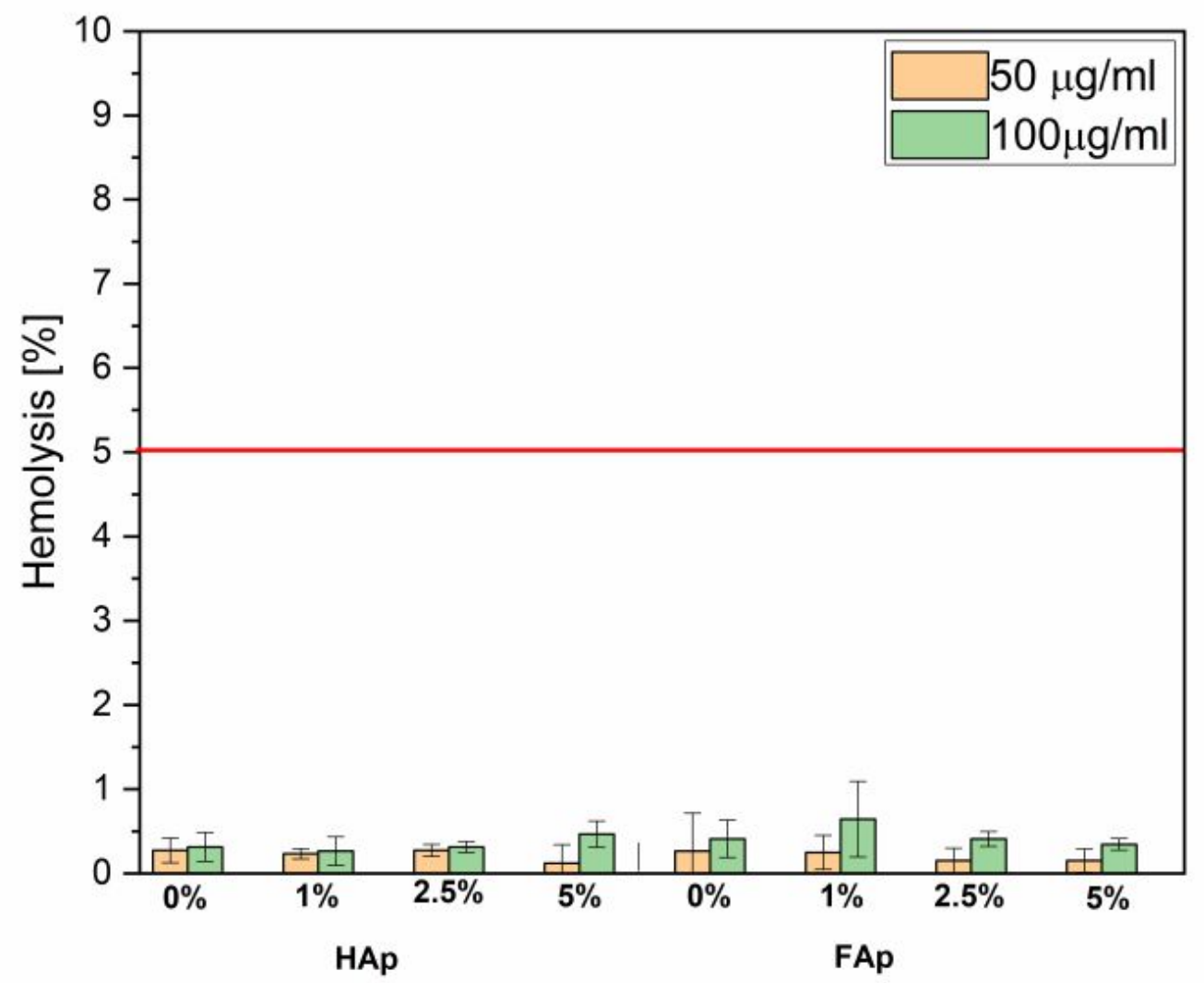

Figure S6. Hemolysis caused by HAp/FAp doped with 1, 2.5 and 5 mol\% of $\mathrm{Ag}^{+}$compared to hemolysis caused by $1 \% \operatorname{SDS}$ (mean $\pm S D, n=3$; all results were statistically significant $(p<0.05)$. The red line indicates the acceptable hemolysis level. 